\title{
Total Synthesis of Saxitoxin and 11-Saxitoxinethanoic Acid
}

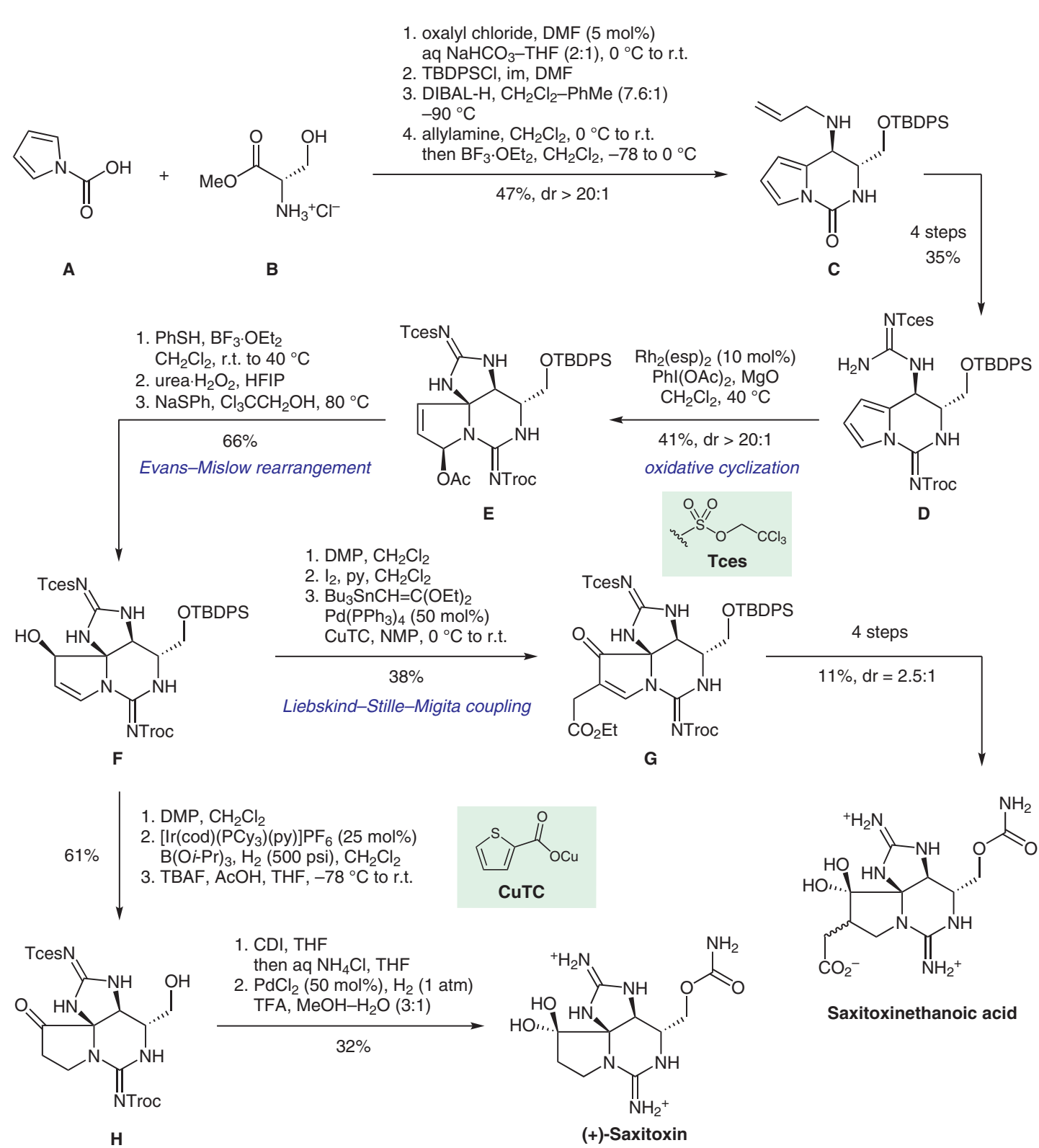

Significance: The authors report the total syntheses of $(+)$-saxitoxin and several structural analogues including saxitoxinethanoic acid. These bisguanidinium natural products have been of high interest due to their extraordinary activity against voltage-gated sodium ion channels.
Comment: Electrophilic aromatic substitution and rhodium-catalyzed oxidative cyclization yield tricyclic system $\mathbf{E}$ from simple starting materials. Clever application of an Evans-Mislow rearrangement gives access to advanced intermediate $\mathbf{F}$ that is used to complete the synthesis of both target structures.

SYNFACTS Contributors: Erick M. Carreira, Felix Pultar 